\title{
An improved methane adsorption model in shale by considering variable adsorbed phase density
}

Xingxing Kong ${ }^{\ddagger 1}$, Hongjun Fan ${ }^{\ddagger *}$, Dianshi Xiao ${ }^{\ddagger 2}$, Pengfei $\mathrm{Mu}^{\ddagger 3}$, Shuangfang $\mathrm{Lu}^{\ddagger 2}$, Shu Jiang $^{14}$, Guohui Chen ${ }^{14}$

${ }^{1}$ CNOOC Research Institute Co. Ltd., Beijing 100028, China

${ }^{2}$ School of Geosciences, China University of Petroleum (East China), Qingdao 266580, China

${ }^{3}$ Bohai Oilfield Research Institute, Tianjin Branch of CNOOC Ltd., Tianjin 300452, China

${ }^{4}$ Key Laboratory of Tectonics and Petroleum Resources of Ministry of Education, School of Earth Resources, China University of Geosciences, Wuhan 430074, China 
Nine shale samples from the Wufeng-Longmaxi Formation in the Sichuan Basin were tested for total organic carbon (TOC), X-ray diffraction (XRD) analysis, low-temperature nitrogen adsorption, and methane isothermal adsorption. The experimental results are shown in Table S1, Figures S1 and S2.

(1) TOC and XRD

The TOC was measured on the CS230 high-frequency infrared carbon-sulfur analyzer produced by the American LECO company. During the experiment, the samples need to be crushed to 100-200 mesh and treated with hydrochloric acid to remove inorganic carbon.

The XRD is to measure the mineral composition of shale samples. The experiment was carried out on a high-resolution X-ray diffractometer of Rigaku Corporation. The samples need to be crushed to more than 200 meshes before the experiment.

(2) Low temperature nitrogen adsorption

This low-temperature nitrogen adsorption was performed on the ASAP 2460 specific surface area and pore size analyzer produced by Micromeritics, USA. First, weigh 3-5g of 60-80 mesh shale powder sample into a sample tube, and dry it under vacuum at $110^{\circ} \mathrm{C}$ for 12 hours. Then weigh the mass of the dried sample, load the sample tube with the dried sample into the system, so that the entire sample tube is immersed in liquid nitrogen $(77 \mathrm{~K})$, and the entire adsorption process is performed below the critical temperature of nitrogen $(126.1 \mathrm{~K})$, so the experimental process is subcritical adsorption. The measurement of the adsorption curve and the desorption curve is automatically completed by the instrument. 
This time the adsorption curve measures 33 pressure points and the desorption curve 23 pressure points. The calculation of the specific surface area uses the BET model ${ }^{1}$.

(3) Methane isotherm adsorption

The methane isotherm adsorption is conducted by gravimetric method. The gravimetric method mainly measures the amount of shale methane adsorbed by the change in the buoyancy of the adsorption phase ${ }^{2}$. The tested sample is a 60-80 mesh powder sample, which should be vacuum dried at $110^{\circ} \mathrm{C}$ for more than 10 hours before the test. The maximum experimental pressure is $28 \mathrm{MPa}$, the number of measurement pressure points for a single experiment is not less than 14 , and the experimental temperature is $60^{\circ} \mathrm{C}$. 
Table S1 The information of the samples

\begin{tabular}{|c|c|c|c|c|c|c|c|c|c|c|c|c|c|}
\hline \multirow[t]{2}{*}{ Sample } & TOC & $\mathrm{S}_{\mathrm{BET}}$ & $\mathrm{V}_{\mathrm{DR}}$ & Quartz & $\begin{array}{l}\text { K- } \\
\text { feldspar }\end{array}$ & Plagioclase & Calcite & Dolomite & Pyrite & Barite & Fluorapatite & Ankerite & Clay \\
\hline & /(\%) & $/\left(\mathrm{m}^{2} / \mathrm{g}\right)$ & $/\left(\mathrm{cm}^{3} / \mathrm{Kg}\right)$ & $/(\%)$ & /(\%) & $/(\%)$ & $/(\%)$ & $/(\%)$ & $/(\%)$ & $/(\%)$ & $/(\%)$ & $/(\%)$ & $/(\%)$ \\
\hline AY1 & 2.10 & 12.13 & 5.78 & 29.9 & 0.8 & 3 & 0.6 & 32.8 & 2.6 & 0 & 0 & 0 & 30.3 \\
\hline BY1 & 4.10 & 18.42 & 8.43 & 34.5 & 0.5 & 3.2 & 0 & 1.2 & 8.3 & 4.6 & 27 & 0.2 & 20.5 \\
\hline BY2 & 2.93 & 14.56 & 6.85 & 53.8 & 1.1 & 3.8 & 5.2 & 4.6 & 3 & 0 & 0 & 0 & 28.5 \\
\hline BY3 & 1.25 & 2.66 & 1.43 & 31.8 & 2.1 & 7.2 & 6.9 & 4.6 & 2.6 & 0 & 0 & 0 & 44.8 \\
\hline CY1 & 0.98 & 7.58 & 3.69 & 36.6 & 1 & 6.6 & 2 & 2.6 & 3.1 & 0 & 0 & 0 & 48.1 \\
\hline CY2 & 2.84 & 17.68 & 8.86 & 46.9 & 1 & 4.3 & 1.6 & 2.7 & 2.7 & 0 & 0 & 0 & 40.8 \\
\hline CY3 & 3.10 & 17.70 & 8.45 & 45.3 & 1.8 & 5.4 & 3.2 & 3.5 & 3.4 & 0 & 0 & 0 & 37.4 \\
\hline CY4 & 2.61 & 13.73 & 6.58 & 53.6 & 1.1 & 5.7 & 2.9 & 5.6 & 2.2 & 0 & 0 & 0 & 28.9 \\
\hline CY5 & 3.90 & 23.65 & 11.55 & 58.7 & 1.1 & 4.5 & 0.2 & 3.6 & 0.3 & 0 & 0 & 0 & 31.6 \\
\hline
\end{tabular}

\footnotetext{
${ }^{1} S_{B E T}$, the total surface area by the BET equation; VDR, the surface area and the micropores volume by the DR equation ${ }^{1}$
} 


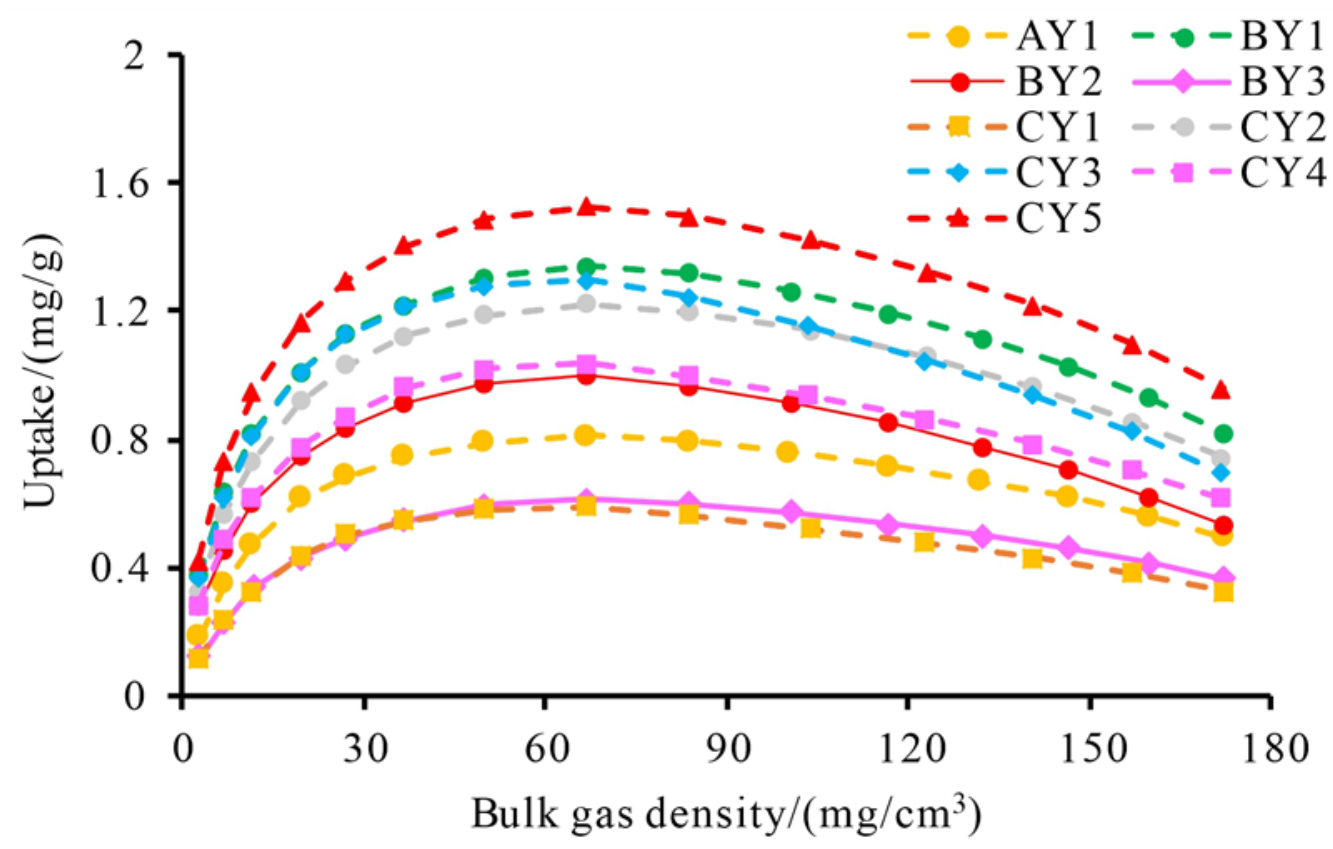

Figure S1 Measured excess adsorption isotherms of methane at 333.15K for the nine samples

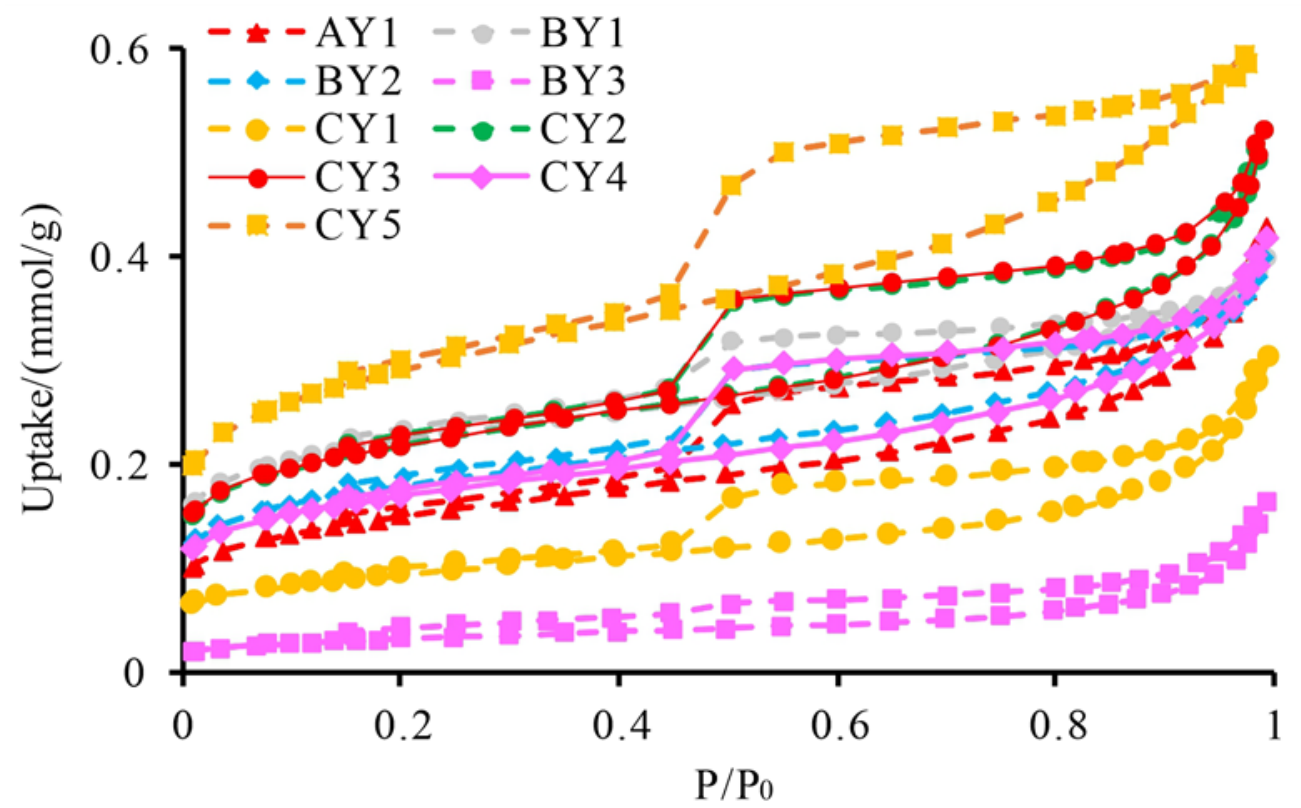

Figure S2 N2 sorption/desorption curves for the nine samples 
In recent years, the Langmuir model (Formula S1) has also been widely used in the study of shale methane isotherm adsorption ${ }^{2-4}$. In order to compare the models more comprehensively, we added the fitting results of the Langmuir model (Formula S1, Table S2). There are many abnormalities in the adsorption phase density obtained by the Langmuir model (the $\rho_{a b s}$ is bigger than $424 \mathrm{mg} / \mathrm{cm}^{3}$, the density of liquid methane at its boiling temperature under $0.1 \mathrm{MPa}^{3}$ ), and the fitted RESM is significantly higher than the VD model and the SDR model. It shows that the fitting stability of SDR model and VD model has been significantly higher than that of the Langmuir model.

$$
n_{e x}=n_{0} \frac{k p}{1+k p}\left(1-\frac{\rho_{g}}{\rho_{a b s}}\right)
$$

where $n_{0}$ represents the maximum absolute adsorption capacity at a given temperature, with units of $\mathrm{mg} / \mathrm{cm}^{3} ; \rho_{a b s}$ is the adsorbed phase density, with unit of $\mathrm{mg} / \mathrm{cm}^{3}$; $\mathrm{k}$ is the Langmuir constant, with units of $\mathrm{Mpa}^{-1} ; \rho_{g}$ is the bulk gas density, with units of $\mathrm{mg} / \mathrm{cm}^{3}$, which can be obtained from NIST's REFPROP program ${ }^{5} ; n_{e x}$ is the excess adsorption, with units mg/g; P is the equilibrium pressure in units of MPa. 
Table S2 Value of fitted parameters of different models

\begin{tabular}{|c|c|c|c|c|c|c|c|c|c|c|c|}
\hline \multirow[b]{2}{*}{ Sample } & \multirow{2}{*}{$\begin{array}{l}\text { Temperature } \\
(\mathrm{K})\end{array}$} & \multicolumn{3}{|c|}{ VD-based } & \multicolumn{2}{|c|}{ SDR-based } & \multicolumn{5}{|c|}{ Langmuir-based } \\
\hline & & $\begin{array}{c}\rho_{f} \\
\left(\mathrm{mg} / \mathrm{cm}^{3}\right.\end{array}$ & $\begin{array}{l}\mathrm{d} \\
\left(\mathrm{cm}^{3} / \mathrm{mg}\right)\end{array}$ & $\begin{array}{c}V_{a} \\
\left(\mathrm{~cm}^{3} / \mathrm{g}\right)\end{array}$ & $\begin{array}{cc} & n_{0} \\
& \\
& \text { RMg/g) }\end{array}$ & $\begin{array}{c}\rho_{a b s} \\
\left(\mathrm{mg} / \mathrm{cm}^{3}\right.\end{array}$ & $\begin{array}{l}\mathrm{D} \\
\left(\mathrm{mol}^{2} / \mathrm{Kj}^{2}\right)\end{array}$ & $\begin{array}{cc} & n_{0} \\
\text { RMSE } & \\
& (\mathrm{mg} / \mathrm{g})\end{array}$ & $\begin{array}{c}\rho_{a b s} \\
\left(\mathrm{mg} / \mathrm{cm}^{3}\right.\end{array}$ & $\begin{array}{l}\mathrm{k} \\
\left(\mathrm{MPa}^{-1}\right)\end{array}$ & RMSE \\
\hline & 300.00 & 127.72 & 0.58306 & 0.00673 & 0.022124 .017 & 655.54 & 0.00856 & $0.01422 \mathrm{nd}^{\mathrm{a}}$ & nd & nd & nd \\
\hline & 303.00 & 129.74 & 0.59240 & 0.00625 & 0.021133 .813 & 659.56 & 0.00836 & 0.01449 nd & nd & nd & nd \\
\hline & 308.00 & 88.95 & 0.48361 & 0.00974 & 0.016883 .507 & 467.17 & 0.00917 & 0.010912 .750 & 5132.06 & 0.72577 & 0.04159 \\
\hline & 318.00 & 82.30 & 0.36899 & 0.01120 & 0.027843 .338 & 419.61 & 0.00942 & 0.020852 .749 & 1372.61 & 0.58683 & 0.04786 \\
\hline & 338.00 & 89.82 & 0.25224 & 0.01086 & 0.019033 .180 & 428.71 & 0.01039 & 0.015462 .631 & 1598.61 & 0.43997 & 0.03122 \\
\hline Alum shale & 358.00 & 73.34 & 0.14209 & 0.01733 & 0.020672 .883 & 318.26 & 0.01009 & 0.021542 .897 & 387.56 & 0.27678 & 0.02807 \\
\hline & 373.00 & 65.67 & 0.10790 & 0.02173 & 0.018162 .528 & 256.82 & 0.01049 & 0.022412 .908 & 249.69 & 0.21934 & 0.02318 \\
\hline & 398.00 & 65.88 & 0.06713 & 0.02727 & 0.015562 .130 & 216.68 & 0.01077 & 0.020852 .991 & 179.05 & 0.14556 & 0.01754 \\
\hline & 423.00 & 67.33 & 0.04320 & 0.03877 & 0.009321 .845 & 169.60 & 0.01129 & 0.019433 .381 & 129.26 & 0.09788 & 0.01023 \\
\hline & 448.00 & 72.75 & 0.02899 & 0.05430 & 0.008191 .547 & 130.37 & 0.01191 & 0.020223 .817 & 98.80 & 0.06730 & 0.00845 \\
\hline & 473.00 & 66.59 & 0.03110 & 0.04710 & 0.012161 .179 & 114.22 & 0.01093 & 0.019582 .956 & 88.20 & 0.06878 & 0.01242 \\
\hline
\end{tabular}




\begin{tabular}{|c|c|c|c|c|c|c|c|c|c|c|c|}
\hline & 308.55 & 69.18 & 0.29931 & 0.00665 & 0.008931 .444 & 338.69 & 0.01107 & 0.007311 .347 & 464.72 & 0.48966 & 0.01721 \\
\hline \multirow[t]{3}{*}{ YC4-04 } & 323.55 & 69.20 & 0.20482 & 0.00767 & 0.009331 .413 & 312.46 & 0.01138 & 0.008591 .400 & 379.51 & 0.36728 & 0.01535 \\
\hline & 338.55 & 68.96 & 0.16891 & 0.00833 & 0.008991 .386 & 298.16 & 0.01107 & 0.009191 .415 & 345.11 & 0.31458 & 0.01386 \\
\hline & 308.55 & 70.57 & 0.28595 & 0.00910 & 0.014831 .994 & 344.91 & 0.01112 & 0.011731 .866 & 473.97 & 0.47302 & 0.02635 \\
\hline \multirow[t]{3}{*}{ YC4-08 } & 323.55 & 69.41 & 0.21713 & 0.00999 & 0.012221 .899 & 317.66 & 0.01116 & 0.011331 .841 & 406.31 & 0.38800 & 0.01938 \\
\hline & 338.55 & 71.03 & 0.15404 & 0.01102 & 0.006481 .830 & 299.86 & 0.01141 & 0.007411 .869 & 353.44 & 0.29923 & 0.01171 \\
\hline & 308.55 & 75.67 & 0.28401 & 0.00745 & 0.014431 .794 & 374.46 & 0.01090 & 0.011191 .636 & 576.95 & 0.47377 & 0.02409 \\
\hline \multirow[t]{3}{*}{ YC4-33 } & 323.55 & 73.33 & 0.22833 & 0.00802 & 0.011601 .689 & 344.68 & 0.01075 & 0.009831 .589 & 484.41 & 0.40093 & 0.01887 \\
\hline & 338.55 & 75.35 & 0.19268 & 0.00801 & 0.010261 .625 & 344.32 & 0.01028 & 0.009301 .544 & 477.82 & 0.34817 & 0.01585 \\
\hline & 308.55 & 74.93 & 0.35886 & 0.00908 & 0.019612 .354 & 387.93 & 0.01013 & 0.014252 .098 & 631.08 & 0.54431 & 0.03518 \\
\hline \multirow[t]{3}{*}{ YC4-47 } & 323.55 & 71.85 & 0.29502 & 0.00950 & 0.017692 .160 & 355.34 & 0.00994 & 0.013961 .977 & 525.07 & 0.46765 & 0.02963 \\
\hline & 338.55 & 69.07 & 0.24324 & 0.01031 & 0.014122 .048 & 324.90 & 0.00981 & 0.011951 .930 & 442.13 & 0.40457 & 0.02341 \\
\hline & 308.55 & 75.91 & 0.57219 & 0.00883 & 0.017442 .710 & 413.78 & 0.00888 & 0.008722 .175 & 2083.75 & 0.80629 & 0.03625 \\
\hline \multirow[t]{2}{*}{ YC4-54 } & 323.55 & 75.17 & 0.42705 & 0.00930 & 0.014622 .573 & 387.73 & 0.00890 & 0.008132 .103 & 1440.41 & 0.65297 & 0.02911 \\
\hline & 338.55 & 83.57 & 0.33988 & 0.00829 & 0.013052 .446 & 413.42 & 0.00852 & 0.007821 .963 & 2940.23 & 0.54864 & 0.02352 \\
\hline YC4-61 & 308.55 & 72.79 & 0.56548 & 0.01009 & 0.020682 .923 & 403.57 & 0.00891 & 0.011632 .439 & 991.03 & 0.75887 & 0.04398 \\
\hline
\end{tabular}




\begin{tabular}{|c|c|c|c|c|c|c|c|c|c|c|c|}
\hline & 323.55 & 68.82 & 0.45821 & 0.01100 & 0.018052 .769 & 363.34 & 0.00882 & 0.010882 .360 & 753.27 & 0.65262 & 0.03666 \\
\hline & 338.55 & 69.50 & 0.37360 & 0.01112 & 0.015902 .644 & 352.26 & 0.00859 & 0.010162 .271 & 720.11 & 0.55974 & 0.03049 \\
\hline & 308.55 & 68.51 & 0.38357 & 0.01177 & 0.019782 .768 & 353.84 & 0.01020 & 0.013652 .513 & 513.41 & 0.56909 & 0.04002 \\
\hline \multirow[t]{3}{*}{ YC4-64 } & 323.55 & 66.23 & 0.30191 & 0.01268 & 0.018642 .597 & 322.96 & 0.01015 & 0.014782 .448 & 422.37 & 0.47113 & 0.03432 \\
\hline & 338.55 & 69.87 & 0.24863 & 0.01213 & 0.014192 .475 & 329.40 & 0.00974 & 0.011382 .323 & 448.97 & 0.40772 & 0.02625 \\
\hline & 308.55 & 67.65 & 0.50397 & 0.01161 & 0.021852 .946 & 366.43 & 0.00940 & 0.014012 .552 & 659.14 & 0.69919 & 0.04488 \\
\hline \multirow[t]{2}{*}{ YC4-65 } & 323.55 & 71.99 & 0.39976 & 0.01104 & 0.018902 .818 & 374.17 & 0.00907 & 0.012152 .429 & 730.60 & 0.58937 & 0.03655 \\
\hline & 338.55 & 73.55 & 0.32352 & 0.01088 & 0.014892 .653 & 366.12 & 0.00884 & 0.009672 .294 & 734.03 & 0.50524 & 0.02830 \\
\hline FC-47 & 393.15 & 65.91 & 0.25643 & 0.00740 & 0.010651 .414 & 302.16 & 0.01034 & 0.010141 .444 & 317.67 & 0.30919 & 0.01829 \\
\hline FC-66 & 393.15 & 73.60 & 0.24365 & 0.01062 & 0.018502 .319 & 345.05 & 0.01006 & 0.018442 .304 & 376.91 & 0.29928 & 0.03165 \\
\hline FC-72 & 393.15 & 74.88 & 0.26089 & 0.01357 & 0.014323 .128 & 357.72 & 0.00981 & 0.014703 .034 & 404.84 & 0.31616 & 0.03016 \\
\hline AY1 & 333.15 & 71.41 & 0.16695 & 0.00725 & 0.004991 .279 & 293.48 & 0.01191 & 0.007331 .418 & 294.49 & 0.26696 & 0.01284 \\
\hline BY1 & 333.15 & 68.51 & 0.22016 & 0.01088 & 0.020402 .054 & 304.01 & 0.01076 & 0.022922 .199 & 309.09 & 0.31536 & 0.03722 \\
\hline BY2 & 333.15 & 66.78 & 0.17871 & 0.00944 & 0.017001 .555 & 274.32 & 0.01184 & 0.020571 .763 & 270.67 & 0.27245 & 0.02795 \\
\hline BY3 & 333.15 & 77.01 & 0.11228 & 0.00620 & 0.004930 .986 & 284.00 & 0.01354 & 0.007621 .185 & 275.34 & 0.20068 & 0.00914 \\
\hline CY1 & 333.15 & 71.31 & 0.13022 & 0.00614 & 0.006020 .951 & 266.39 & 0.01341 & 0.003701 .108 & 263.21 & 0.22914 & 0.00347 \\
\hline
\end{tabular}




\begin{tabular}{llllllllllllll} 
CY2 & 333.15 & 69.39 & 0.19353 & 0.01046 & 0.015651 .895 & 297.16 & 0.01127 & 0.01890 & 2.068 & 299.20 & 0.29172 & 0.03028 \\
CY3 & 333.15 & 63.40 & 0.21762 & 0.01193 & 0.012352 .008 & 271.05 & 0.01128 & 0.01543 & 2.190 & 272.76 & 0.31894 & 0.02775 \\
CY4 & 333.15 & 67.11 & 0.20863 & 0.00889 & 0.013611 .591 & 289.95 & 0.01114 & 0.014571 .726 & 292.81 & 0.30722 & 0.02432 \\
CY5 & 333.15 & 68.30 & 0.23308 & 0.01210 & 0.017212 .333 & 307.47 & 0.01057 & 0.01949 & 2.457 & 316.48 & 0.33184 & 0.03708 \\
\hline
\end{tabular}

${ }^{a}$ nd: no data. During the fitting process, the global optimal solution that meets the physical meaning cannot be found. 


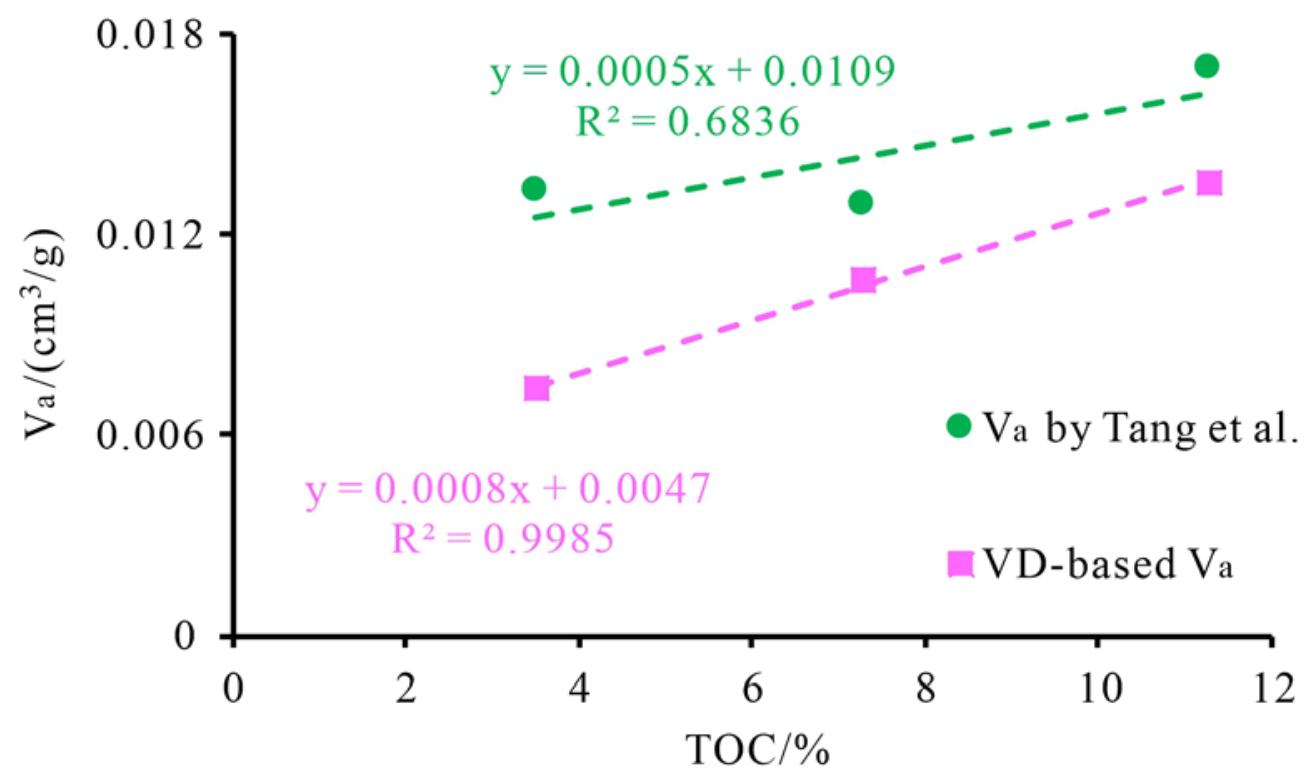

Figure S3 The relationship between TOC with $V_{a}$ obtained by different model

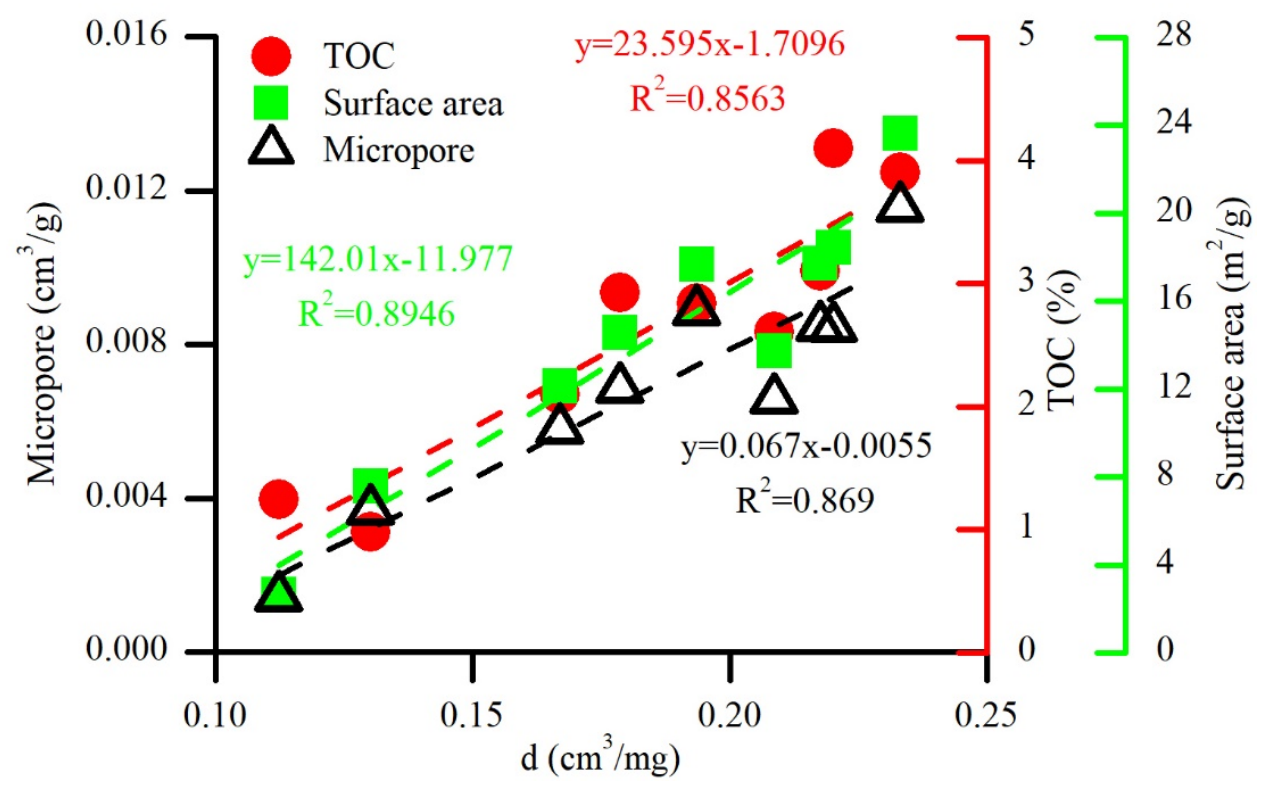

Figure S4 Relationship between d with micropore, surface area and TOC 


\section{Reference}

1. Sing, K. S., Reporting physisorption data for gas/solid systems with special reference to the determination of surface area and porosity (Recommendations 1984). Pure and applied chemistry 1985, 57, (4), 603-619.

2. Zhou, S.; Xue, H.; Ning, Y.; Guo, W.; Zhang, Q., Experimental study of supercritical methane adsorption in Longmaxi shale: Insights into the density of adsorbed methane. Fuel 2018, 211, 140-148.

3. Tian, H.; Li, T.; Zhang, T.; Xiao, X., Characterization of methane adsorption on overmature Lower Silurian-Upper Ordovician shales in Sichuan Basin, southwest China: Experimental results and geological implications. International Journal of Coal Geology 2016, 156, 36-49.

4. Gai, H.; Li, T.; Wang, X.; Tian, H.; Xiao, X.; Zhou, Q., Methane adsorption characteristics of overmature Lower Cambrian shales of deepwater shelf facies in Southwest China. Marine and Petroleum Geology 2020, 120, 104565.

5. Lemmon, E.; Huber, M.; McLinden, M., NIST Standard Reference Database 23, NIST Reference Fluid Thermodynamic and Transport Properties, REFPROP, version 9.0. Standard Reference Data Program 2010. 